\title{
Random controls: A rejoinder
}

\author{
WILLIAM F. PROKASY \\ University of Utah, Salt Lake City, Utah 84112
}

\begin{abstract}
Furedy, Poulos, and Schiffman (1975) have made a conclusion in direct contradiction to available data in skin conductance conditioning which show clearly that a stimulus designated to be random was, in fact, random with respect to an unconditioned stimulus. It was also pointed out that the overlap criterion does illustrate the weakness of the Toronto studies; that the random stimulus in the Prokasy, Williams, Kumpfer, and Lee (1973) paper was not excitatory; and that controlled studies for at least a decade have shown the first-interval response to be associative.
\end{abstract}

There are four general points to be made in response to the Furedy, Poulos, and Schiffman paper (1975a) paper. First, these authors allege that I have not dealt with the logical inadequacies which they feel characterize the design of the Prokasy, Williams, Kumpfer, and Lee (1973) paper. Elsewhere (Furedy, Poulos, \& Schiffman (1975b) they correctly point out that removing all pre programmed randomly presented unconditional stimuli (UCs) other than those which occur in the presence of a signal makes that signal an excitatory conditioned stimulus (CS). They then conclude by (incorrect) analogy that the elimination of randomly generated USs which are located in the vicinity of a signal which is to become a CS- means that a second signal which is supposed to remain random with respect to US occurrence becomes excitatory. They state that this procedure makes the conditional likelihood of a US in the presence of an RS greater than the conditional likelihood of a US in the absence of an RS. Table 1 in Prokasy (1975) provides the actuarial facts of the Prokasy et al. (1973) experiment and it shows unequivocally that the Furedy et al. (1975a, b,) conclusion is incorrect.

Second, Furedy et al. (1974a) state that the "overlap criterion" for judging randomness leads to paradoxical consequences in that, as one example, what would otherwise be considered a CS+ in trace conditioning would have to be defined as a CS- because there is no overlap of CS and US. On that basis, they reject the tabled data in Prokasy (1975), which show (1) that the Prokasy et al. (1973) study did not have an inadvertently excitatory US based on conditional likelihoods and (2) that the Toronto studies do not admit of conclusions concerning whether or not CS- and RS lead to differential performance since the RS was more like a CS- than an RS. It is unnecessary to deal here with the "paradoxes" claimed by Furedy et al. other than to point out that at no time was the index of overlap argued to be a genera! criterion for determining what is or is not a $\mathrm{CS}+$ or $\mathrm{CS}_{-}$, and that should be clear in the paper's context. How-

Supported by NIMH Grant MH-15353. ever, my intention is immaterial because whatever might be thought about the "paradoxes" has absolutely no bearing on the actuarial facts of the studies in question. The RS either was or was not random with respect to the US, and the tabled values provided by Prokasy (1975) will permit the readers to judge whether or not the two points made above about those values are reasonable.

Third, in no sense did Furedy et al. (1975, a,b) show that the RS in the Prokasy et al. (1973) study was excitatory on the basis of expected probabilities. They simply pointed out that, by their calculation, on $39 \%$ of the RS trials a US occurred within $13 \mathrm{sec}$ of RS onset. That fact cannot be employed to conclude that the RS was excitatory, inhibitory, or neutral. Such a conclusion can rest only on what the data outcomes were. Because first-interval and second-interval responses did not yield the same data outcomes, i.e., because there was a failure of internal consistency when alternative measures of conditioning were employed, Prokasy et al. (1973) concluded that it was " ... difficult to interpret the CSs as acquiring general excitatory or inhibitory properties." It was also noted that because of the response-eliciting properties of signal onset the second-interval response might provide a clearer measure of excitatory and inhibitory influences. The result was that second-interval RS performance equaled performance obtained with time samples taken in the absence of stimulation, a result which suggests that the RS was not excitatory with respect to the background.

Finally, there are several matters of interpretation. I agree with Furedy et al. (1975a) that the readers might best judge those matters for themselves. One issue, however, does merit some amplification. My interpretation is that the associative status of the first-interval response, within conventional control procedure boundaries (see, e.g., Prokasy \& Kumpfer, 1973), has not been in doubt for at least 10 years. This is an operational position: contingencies such as pairing between CS and US produce a greater first-response frequency than do control treatments. The nature of the response mechanism (e.g., a "newly acquired" response or a sensitized 
orienting response) is important for some reasons, but is immaterial for determining associative influences provided that appropriate controls can permit the conclusion that a pairing effect exists. In citing Dengerink and Taylor (1971), the purpose was to cite a paper with references to older research which permitted the conclusion that the first-interval response is associative. How ambivalent those authors were about the associative status of the first-interval response is unknown to me, but it may be worth pointing out that Furedy et al. (1975a) neglected to quote the following: first-interval responses "tend to habituate, appear to be orienting responses, and, under certain circumstances, they are conditional" (Dengerink \& Taylor, 1971, abstract); and "... results confirm the hypothesis that ... [first-interval] ... responses are conditional and further indicate that the conditional nature of these responses can be maintained over a large number of trials" (Dengerink \& Taylor, 1971 , p. 351).

\section{REFERENCES}

Dengerink, J. A., \& Taylor, S. P. Multiple responses with differential properties in delayed galvanic skin response conditioning: A review. Psychophysiology, 1971, 8, 348-360.

Furedy, J. J., Poulos, C. X.. \& Schiffman, K. Logical problems with Prokasy's assessment of contingency relations in classical skin conductance conditioning. Behavior Research Methods \& Instrumentation, 1975b-7,521-523.

Furedy, J. J., Poulos, C. X., \& Schiffman, K. Contingency theory and classical autonomic excitatory and inhibitory conditionlng: Some problems of assessment and interpretation. Psychophysiology, 1975b, 12,98-105.

Prokasy, W. F. Random control procedures in classical skin conductance conditioning. Behavior Research Methods \& Instrumentation, $1975,7,516-520$.

Prokasy, W. F., \& Kumpfer, K. L. Classical conditioning. In W F. Prokasy and D. C. Raskin (Eds.), Electrodermal activity in psychological research. New York: Academic Press, 1973.

Prokasy, W. F., Williams, W. C., Kumpfer, K. L., Lee, W. Y., \& Jensen, W. R. Differential SCR conditioning with two con trol baselines: Random signal and signal absent. Psychophysiology, $1973,10,145.153$.

(Received for publication August 10, 1975; accepted August 25, 1975.) 